\title{
Neurotoxicities in the treatment of HIV between dolutegravir, rilpivirine and dolutegravir/rilpivirine: a meta-analysis
}

\author{
Anthony Allen Reeves @ ${ }^{1}$ Andrea V Fuentes, ${ }^{1}$ Joshua Caballero, ${ }^{1}$ Jennifer E Thomas, ${ }^{1}$ \\ Juan F Mosley II, ${ }^{1}$ Catherine Harrington ${ }^{2}$
}

\begin{abstract}
- Additional material is published online only. To view, please visit the journal online (http://dx.doi.org/10.1136/ sextrans-2020-054821).

1 Department of Clinical and Administrative Sciences, Larkin University College of Pharmacy, Miami, Florida, USA

'Lloyd L Gregory School of Pharmacy, Palm Beach Atlantic University, West Palm Beach, Florida, USA
\end{abstract}

\section{Correspondence to} Dr Anthony Allen Reeves, Department of Clinical and Administrative Sciences, Larkin University College of Pharmacy, Miami, FL 33169, USA; a. allenreeves13@gmail.com

Received 3 October 2020 Revised 4 January 2021 Accepted 7 January 2021 Published Online First 29 March 2021

\section{ABSTRACT}

Objective To assess the risk of neuropsychiatric adverse effects (ie, depression, anxiety, insomnia, dizziness, suicidal behaviour) among patients treated with rilpivirine, dolutegravir and dolutegravir/rilpivirine. Design This is a systematic review and meta-analysis of randomised controlled trials. Quality of evidence was assessed using Jadad scoring system.

Data sources Three electronic databases were searched for available publications up to 1 May 2020. Searches included relevant studies, trial registers, conference proceeding abstracts and grey literature. Inclusion criteria Randomised controlled trials with data focused on adult participants (ie, 18 years of age or older) receiving dolutegravir $50 \mathrm{mg}$, rilpivirine $25 \mathrm{mg}$ or combination of dolutegravir $50 \mathrm{mg} /$ rilpivirine $25 \mathrm{mg}$ once daily.

Results Twenty studies with a minimum duration of 48 weeks and average Jadad score of 4 were included ( $n=10$ 998). Primary objective demonstrated a relative risk (RR) synergistic effect on depressive symptoms for dolutegravir/rilpivirine ( $R R=2.82 ; 95 \% \mathrm{Cl}(1.12$ to 7.10$))$ when compared with dolutegravir $(\mathrm{RR}=1.10 ; 95 \% \mathrm{Cl}$ (0.88 to 1.38$)$ ) and rilpivirine ( $R R=1.08 ; 95 \% \mathrm{Cl}(0.80$ to 1.48)). Secondary objectives showed no difference between dolutegravir, rilpivirine and dolutegravir/ rilpivirine to efavirenz. Additionally, excluding efavirenz studies, dolutegravir and dolutegravir/rilpivirine yielded increased depression ( $R R=1.34 ; 95 \% \mathrm{Cl}$ (1.04 to 1.74)). Conclusion The combination of dolutegravir/rilpivirine appears to increase the risk of depressive symptoms. Despite the increase, the clinical significance is unknown and needs further study. Additionally, neurotoxicity risk appears similar between dolutegravir, rilpivirine and dolutegravir/rilpivirine antiretroviral therapy when compared with efavirenz-based antiretroviral therapy.

\section{INTRODUCTION}

Approximately 37.9 million people worldwide live with HIV and about 25.4 million people use antiretroviral therapy (ART) to manage their infection. ${ }^{1}$ HIV compromises the immune system by attacking CD4 white blood cells. Without ART, HIV can progress to AIDS, defined as a CD4 count $<200$ cells $/ \mathrm{mm}^{3}$, increasing the risk for opportunistic infections. ${ }^{2}$

Patients need to be at least $80 \%$ adherent to their ART to continuously suppress the progression of $\mathrm{HIV}^{2}{ }^{2}$ Reasons for non-adherence include intolerable side-effects, economic/social status, fear of perceived stigma and depression. Depression is a vital concern due to the clinical presentations of amotivation, sleep disturbances, apathy and suicidal ideation. $^{3}$ People living with HIV who exhibit amotivation and other depressive-related symptoms are more likely to be non-adherent and tend to have a poor outlook on life. ${ }^{3}$ Some antiretrovirals (ARVs) are known to cause neuropsychiatric adverse effects such as depression. ${ }^{4}$ Identifying ARVs associated with neuropsychiatric adverse effects or neurotoxicities can assist in properly assessing potential risk factors that can lead to non-adherence and discontinuation due to intolerance; thereby ensuring practitioners provide the most comprehensive care in the management of HIV.

ART is designed to attack the life cycle of HIV preventing its replication. It is usually composed of three-drug regimens with each drug hindering a step in the replication cycle. Manufacturers currently produce two/three drug combinations to improve adherence and decrease the burden of adverse effects. Efavirenz, a component in one of the first Food and Drug Administration (FDA)approved three-drug single tablet regimens, has traditionally been used in ART. However, it is being used less frequently in clinical guidelines such as the US Department of Health and Human Services AIDSinfo due to its extensive neuropsychiatric adverse effects. ${ }^{5}$ Newer agents such as a two-drug combination composed of dolutegravir and rilpivirine have become an alternative regimen due to perceived less neurotoxic adverse effects. Dolutegravir is a commonly prescribed ART that has become a first-line agent in the USA and Europe with over $50 \%$ of regimens containing this agent. ${ }^{6}$ The WHO has even classified dolutegravir as an Essential Medicine for developing countries. ${ }^{6}$ Dolutegravir is an integrase strand transfer inhibitor (INSTI) that prevents the integration of viral DNA into host cell DNA. ${ }^{7}$ Rilpivirine is a non-nucleoside reverse transcriptase inhibitor (NNRTI) that blocks HIV reverse transcriptase. ${ }^{8}$ As a result, both medications act synergistically to suppress viral replication inhibiting two different pathways of the HIV replication cycle. However, the ARV combination of dolutegravir and rilpivirine has a warning for depressive disorders and other neuropsychiatric adverse effects based on the rilpivirine component. ${ }^{9}$ Other neuropsychiatric adverse effects include anxiety, insomnia, dizziness and suicidal behaviours. ${ }^{10}$ Rilpivirine is documented to exacerbate 
depressive symptoms and other central nervous system (CNS) toxicities, thereby providing caution when prescribing rilpivirine to patients with a history or diagnosis of depression. ${ }^{89}$ While dolutegravir has been linked to neurotoxicities as well, at this time it does not warrant the same precaution as rilpivirine. ${ }^{78}$ Additionally, it is unknown if these agents differ in neuropsychiatric adverse effects compared with non-preferred efavirenzbased treatments. Therefore, the primary objective of this study was to evaluate the risk of neurotoxicities (ie, depression, anxiety, insomnia, dizziness, suicidal behaviour) among patients treated with ARVs rilpivirine, dolutegravir and dolutegravir/rilpivirine. The secondary objective was to compare the risk between the aforementioned agents and efavirenz-based therapy.

\section{METHOD}

A systematic review of literature was conducted to identify studies observing participants taking an oral tablet of either dolutegravir, rilpivirine or the combination of dolutegravir/ rilpivirine in the treatment of HIV. MEDLINE via PubMed, AIDSinfo.nih.gov and ClinicalTrials.gov medical databases were searched for available publications up to 1 May 2020. The keyword search terms used were the generic, brand and scientific names for the following medications "rilpivirine/Edurant/ TMC278", "dolutegravir/Tivicay", "rilpivirine and dolutegravir/ Juluca". These terms were paired with "toxicity" and/or "neurotoxicity" (eg, dolutegravir toxicity). Data were extracted independently by two researchers (AAR, AVF) and reviewed by four peers (JC, JET, JFM, CH) for quality analysis. All searches were limited to only include English-language publications. Adverse event data were collected from ClinicalTrials.gov from both the serious adverse events and other adverse events sections and combined in the data analysis.

Only randomised controlled trials (RCTs) were included. Masking could either be open labelled or double blinded. Studies were included if they involved adult participants 18 years of age or older who received at least one dose of dolutegravir $50 \mathrm{mg}$, rilpivirine $25 \mathrm{mg}$ or combination of dolutegravir $50 \mathrm{mg} /$ rilpivirine $25 \mathrm{mg}$ once daily. All participants, except those receiving dolutegravir/rilpivirine, must have been on a guideline-based HIV backbone regimen. The HIV backbone regimen was defined as two nucleoside reverse transcriptase inhibitors (NRTIs), which are combined with a third ARV HIV drug from either the NNRTIs, protease inhibitors or INSTIs drug class. ${ }^{11}$ Any duration of adverse drug events reported was included. Exclusion criteria consisted of observational studies (eg, cohorts), studies involving paediatric/adolescents, studies withdrawn or terminated early, studies not reporting adverse events data, using non-specified doses and if adverse event data reported were inconclusive. Inconclusive was defined as a study using the same drug in both treatment and control arms.

The studies were evaluated for their neuropsychiatric adverse events data specifically depression, anxiety, insomnia, dizziness and suicidal behaviour. Due to an overlap of certain related adverse events identified, the events were classified under their respective associated main neuropsychiatric adverse effect being analysed. Depression was defined as itself, depressed mood, major depression, low mood, adjustment disorder with depressed mood, persistent depressive disorder, seasonal affective disorder and sadness. Anxiety was defined as itself, nervousness, panic attacks and panic reactions. Insomnia was defined as itself, restlessness and difficulty sleeping. Dizziness was defined as itself and lightheadedness. Suicidal behaviour was defined as itself, suicide attempt and suicide ideation.
The quality of the RCTs was assessed using the Jadad scoring method. Jadad score criteria were based on randomisation, generated sequence of randomisation, type of blinding, and description of withdrawals and dropouts. ${ }^{12}$ The scoring system ranged from a scale of $0-5$, with 5 being the highest quality of research design. Two reviewers (AAR, AVF) assessed and provided initial Jadad scores for the included articles. Three reviewers then independently assessed the initial Jadad scores (JC, JET, JFM). If any discrepancies in scoring were identified, a Delphi technique was employed with one author $(\mathrm{CH})$ as the facilitator.

A meta-analysis was conducted to evaluate rates of neuropsychiatric adverse effects. Risk ratios (RRs) were calculated by using the comparison of rilpivirine, dolutegravir and dolutegravir/rilpivirine versus other ART. A primary analysis was conducted using all included studies without exclusions. A subanalysis of RRs was then conducted excluding efavirenz due to its FDA-recognised warnings and documented reports of neuropsychiatric adverse effects. ${ }^{114}$ Review Manager (RevMan) V.5.3 software was used for data analyses to determine the adverse drug event RRs. ${ }^{15}$ RevMan V.5.3 RRs were computed using dichotomous data type, Mantel-Haenszel statistical method, random effects analysis mode and a 95\% CI. All results were crossed checked by JC, JET, JFM and CH.

\section{RESULTS}

The medical database searches identified a total of 71 studies (figure 1). The initial screening of study citations excluded 10 studies due to study design, withdrawal and early termination. Abstracts were then screened for relevance and redundancy excluding an additional 17 studies. A total of 44 studies were fully assessed and 24 were excluded due to the study population, inconclusive adverse event data and lack of neurotoxicity adverse event data. A total of 20 studies met the inclusion criteria to be included in the meta-analysis. ${ }^{16-35}$ The results include 5 studies for rilpivirine, ${ }^{16-20} 14$ studies for dolutegravir ${ }^{21-34}$ and 1 study for dolutegravir/rilpivirine. ${ }^{35}$

The total number of represented patients was 10988 with the majority being male. One study, Orrell et al, had an all-female population. ${ }^{26}$ Out of the 20 studies, 8 used ART-experienced participants and 12 used ART-naïve participants (online supplemental table S1). The shortest duration of reported adverse drug events was 24 weeks by Trottier et al $(\mathrm{n}=1)$ and the longest was 164 weeks by NCT00543725 THRIVE trial $(n=1)$. The

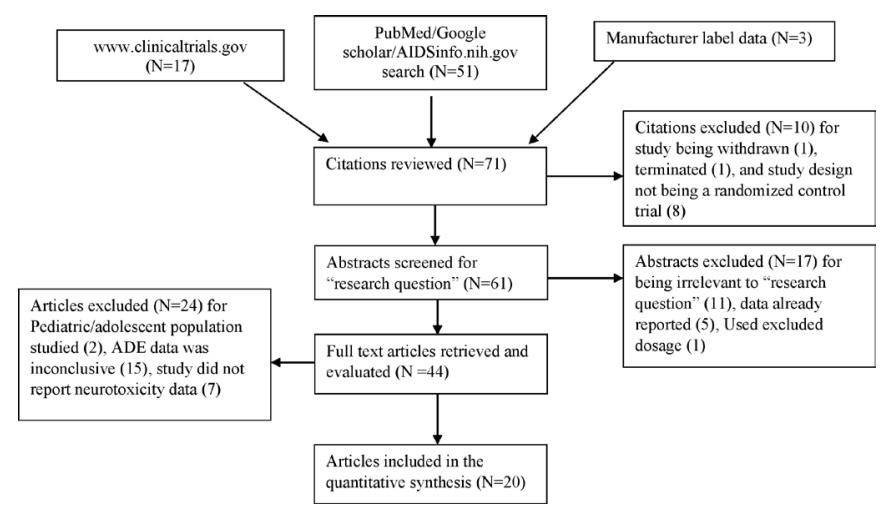

Figure 1 Selection process for RCTs of dolutegravir, rilpivirine and dolutegravir/rilpivirine. Initially included 71 possible citations. We excluded 51 articles/RCTs due to not matching inclusion criteria. The final analysis included 20 articles/RCTs. ADE, adverse drug event; RCTs, randomised controlled trials. 
average study Jadad score was 4. Eight studies used efavirenz as a comparator.

\section{Primary outcome: risk of neurotoxicities}

Analysis RR of depression versus ARV comparators

A total of 15 studies observed and reported incidences of depression and were included in the combined analysis (figure 2). However, five studies ${ }^{18} 27283032$ did not evaluate the risk of depression. The RR of depression from the combined data of rilpivirine, dolutegravir and dolutegravir/rilpivirine against ARV comparators was not statistically significant $(\mathrm{RR}=1.13,95 \% \mathrm{CI}$ (0.94 to 1.36$), p=0.19)$. Dolutegravir/rilpivirine had the highest $\mathrm{RR}(\mathrm{RR}=2.82,95 \% \mathrm{CI}(1.12$ to 7.10$), \mathrm{p}=0.03)$. The $\mathrm{RR}$ for dolutegravir $(\mathrm{RR}=1.10,95 \% \mathrm{CI}(0.88$ to 1.38$), \mathrm{p}=0.39)$ and rilpivirine $(\mathrm{RR}=1.08,95 \% \mathrm{CI}(0.80$ to 1.48$), \mathrm{p}=0.60)$ was not statistically significant.

\section{Subanalysis RR of depression excluding efavirenz versus ARV comparators}

A total of 20 studies were included in the meta-analysis. A subanalysis excluding studies involving efavirenz was conducted. A total of eight studies were identified using efavirenz ${ }^{16-212327}$ as the comparator arm. Out of the 12 remaining studies, only 8 of them observed and reported incidences of depression (figure 3). When excluding efavirenz, the combined RR becomes statistically significant in the outcome of depression $(\mathrm{RR}=1.34,95 \%$ CI (1.04 to 1.74$), p=0.02)$. The RR for dolutegravir/rilpivirine did not change from 2.82; however, the 95\% CI slightly shortened to $95 \%$ CI (1.12 to 7.07). The RR for dolutegravir changed to $(\mathrm{RR}=1.26,95 \% \mathrm{CI}(0.96$ to 1.65$), \mathrm{p}=0.09)$.

\section{Analysis RR of anxiety, insomnia, dizziness and suicidal behaviour} versus ARV comparators

The total number of studies analysed for the other neurotoxicities were anxiety $(n=13)$, insomnia $(n=17)$, dizziness $(n=15)$

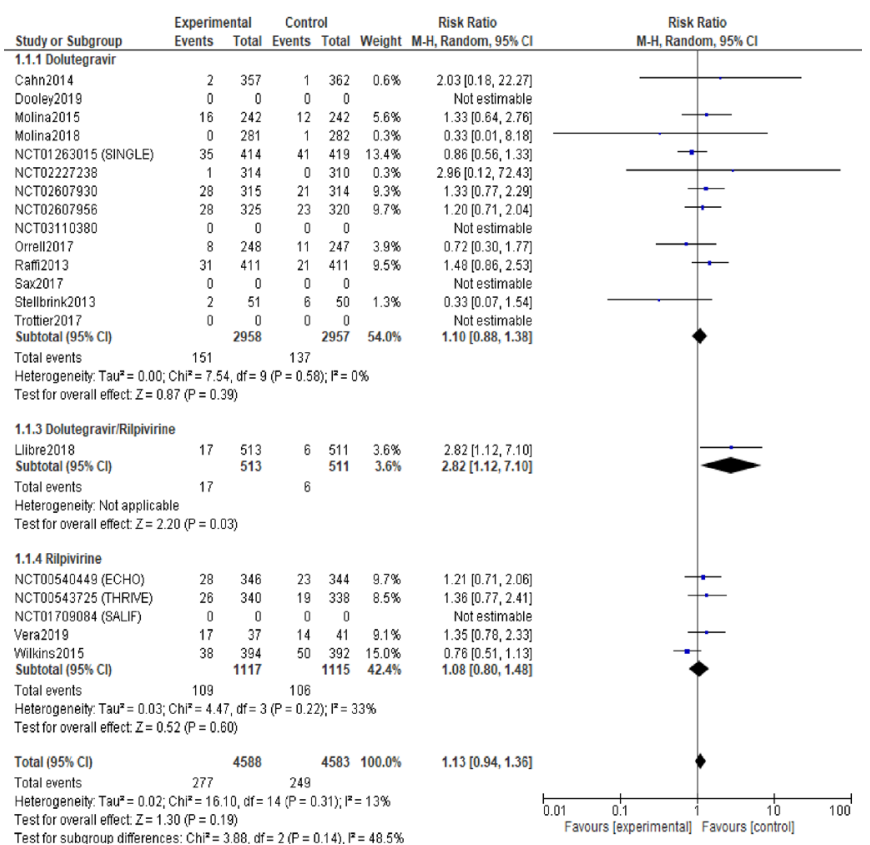

Figure 2 Risk ratio of depression (ART comparators). Forest plot risk ratio estimates with a $95 \% \mathrm{Cl}$ of the association between depression risk and rilpivirine, dolutegravir or the combination of dolutegravir/ rilpivirine administration. ART, antiretroviral therapy.

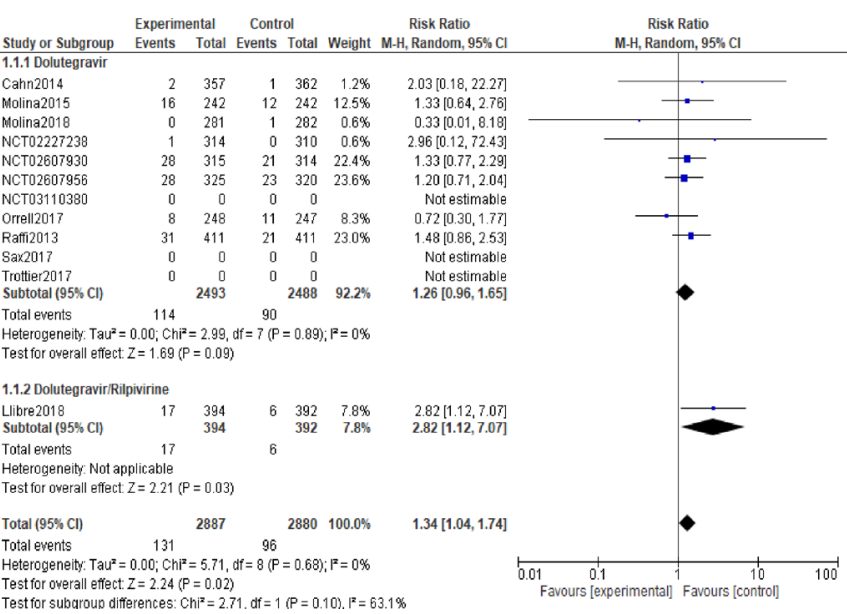

Figure 3 Risk ratio of depression (ART comparators excluding efavirenz). Forest plot risk ratio estimates with a $95 \% \mathrm{Cl}$ of the association between depression risk and rilpivirine, dolutegravir or the combination of dolutegravir/rilpivirine administration excluding studies involving efavirenz. ART, antiretroviral therapy.

and suicidal behaviour $(n=15)$ (table 1$)$. The highest RR was in dizziness with dolutegravir/rilpivirine $(\mathrm{RR}=20.92,95 \% \mathrm{CI}$ (1.23 to 356.03$), p=0.04)$. The lowest and significant $R R$ was in dizziness with rilpivirine $(\mathrm{RR}=0.35,95 \% \mathrm{CI}(0.28$ to 0.43$)$, $\mathrm{p}<0.00001)$.

Subanalysis RR of anxiety, insomnia, dizziness and suicidal behaviour excluding efavirenz versus ARV comparators

There were no significant changes in anxiety, insomnia and suicidal behaviour that was observed when including efavirenz in the analysis. However, dizziness became not statistically significant due to its $\mathrm{p}$ value and $95 \% \mathrm{CI}$ increasing when excluding efavirenz for dolutegravir and the combined analysis $(\mathrm{RR}=1.12$, $95 \% \mathrm{CI}(0.85$ to 1.48$), \mathrm{p}=0.43)$ and $(\mathrm{RR}=1.23,95 \% \mathrm{CI}(0.94$ to 1.62$), \mathrm{p}=0.14$ ), respectively (table 2 ).

\section{Secondary outcome: compare the risk against efavirenz- based therapies}

There has not been a study comparing efavirenz against the combination of dolutegravir and rilpivirine. We can only analyse dolutegravir and rilpivirine individually against efavirenz. The only statistically significant neuropsychiatric adverse effect was dizziness. In all, dizziness RR was less than one group for dolutegravir $(\mathrm{RR}=0.28,95 \% \mathrm{CI}(0.21$ to 0.38$), \mathrm{p}<0.00001)$, rilpivirine $(\mathrm{RR}=0.35,95 \% \mathrm{CI}(0.28$ to 0.43$), \mathrm{p}<0.00001)$ and combined analysis $(\mathrm{RR}=0.33,95 \% \mathrm{CI}(0.28$ to 0.39$)$, $\mathrm{p}<0.00001)$. Efavirenz has a higher risk of causing dizziness than dolutegravir and rilpivirine. All other neurotoxicities were not statistically significant (table 3 ).

\section{Discontinuation rate of included RCTs}

Out of the 20 included studies, 12 reported discontinuation due to drug-related adverse effects. Only Llibre et al, Molina et al, Orrell et al and Stellbrink et al provided reasons for discontinuation (table 4).

\section{Comparing neuropsychiatric adverse effects with other} adverse drug-related events reported

Depression was the most frequent neuropsychiatric adverse effect to occur while suicidal behaviour was the least. The most 
Table 1 Risk ratios of neurotoxicities including all RCTs

\begin{tabular}{|c|c|c|c|c|c|c|}
\hline Adverse event & Drug(s) & Risk ratio & $95 \% \mathrm{Cl}$ & $P$ value & Studies included & $\mathrm{I}^{2} \%$ \\
\hline \multirow[t]{4}{*}{ Depression } & Dolutegravir & 1.1 & 0.88 to 1.38 & 0.39 & 10 & 0 \\
\hline & Dolutegravir/rilpivirine & 2.82 & 1.12 to 7.10 & 0.03 & 1 & $\mathrm{~N} / \mathrm{A}$ \\
\hline & Rilpivirine & 1.08 & 0.8 to 1.48 & 0.6 & 4 & 33 \\
\hline & Combined & 1.13 & 0.94 to 1.36 & 0.19 & 15 & 13 \\
\hline \multirow[t]{4}{*}{ Anxiety } & Dolutegravir & 0.94 & 0.74 to 1.20 & 0.61 & 9 & 1 \\
\hline & Dolutegravir/rilpivirine & 1.37 & 0.56 to 3.38 & 0.49 & 1 & $\mathrm{~N} / \mathrm{A}$ \\
\hline & Rilpivirine & 0.68 & 0.35 to 1.34 & 0.27 & 3 & 75 \\
\hline & Combined & 0.88 & 0.69 to 1.13 & 0.32 & 13 & 34 \\
\hline \multirow[t]{4}{*}{ Insomnia } & Dolutegravir & 1.18 & 0.93 to 1.48 & 0.17 & 12 & 24 \\
\hline & Dolutegravir/rilpivirine & 1.69 & 0.78 to 3.65 & 0.18 & 1 & $\mathrm{~N} / \mathrm{A}$ \\
\hline & Rilpivirine & 1 & 0.71 to 1.41 & 1 & 4 & 62 \\
\hline & Combined & 1.12 & 1 to 1.41 & 0.24 & 17 & 39 \\
\hline \multirow[t]{4}{*}{ Dizziness } & Dolutegravir & 0.57 & 0.43 to 0.69 & $<0.00001$ & 9 & 83 \\
\hline & Dolutegravir/rilpivirine & 20.92 & 1.23 to 356.03 & 0.04 & 1 & $\mathrm{~N} / \mathrm{A}$ \\
\hline & Rilpivirine & 0.35 & 0.28 to 0.43 & $<0.00001$ & 5 & 0 \\
\hline & Combined & 0.47 & 0.41 to 0.53 & $<0.00001$ & 15 & 79 \\
\hline \multirow[t]{4}{*}{ Suicidal behaviour } & Dolutegravir & 0.93 & 0.56 to 1.57 & 0.8 & 11 & 0 \\
\hline & Dolutegravir/rilpivirine & 2.99 & 0.31 to 28.63 & 0.34 & 1 & N/A \\
\hline & Rilpivirine & 1.32 & 0.36 to 4.8 & 0.67 & 3 & 17 \\
\hline & Combined & 1.02 & 0.64 to 1.62 & 0.93 & 15 & 0 \\
\hline
\end{tabular}

N/A, not applicable; RCTs, randomised controlled trials.

common other drug-related adverse effects reported were diarrhoea, nasopharyngitis, upper respiratory tract infection, headache, nausea and vomiting (online supplemental table S2).

\section{DISCUSSION}

The US Department of Health and Human Services HIV/ AIDS guidelines recommend an INSTI-based regimen, usually combined with two NRTIs, as a first-line treatment option in most individuals living with $\mathrm{HIV}^{5}$ A two-drug regimen containing dolutegravir/lamivudine is also considered a firstline option for patients without HIV RNA >500000 copies/ $\mathrm{mL}$, hepatitis $\mathrm{B}$ coinfection or in whom treatment will be started prior to the results of HIV genotypic resistance testing. ${ }^{5}$ It is important to understand the possible side-effects of dolutegravir due to its standing as a component included in the majority of first-line treatment options.
The mechanism for which dolutegravir induces neurotoxicity is not clearly defined. The neurotoxic adverse effects are postulated to be due to dolutegravir's ability to penetrate the blood-brain barrier through passive diffusion. ${ }^{36} 37$ Other data propose dolutegravir may alter the tight junctions of the bloodbrain barrier and cause neuroinflammation. ${ }^{6}$ Regardless of the mechanism, dolutegravir has shown to accumulate in cerebral spinal fluid and based on animal studies, it is proposed that dolutegravir accumulation in the CNS may cause oxidative stress altering neuronal homeostasis. ${ }^{36}$ A study by Yagura et $a l^{39}$ concluded dolutegravir plasma concentrations should be monitored because an increase in plasma concentration of dolutegravir was positively correlated to an increase of neurotoxic side-effects among their patients. ${ }^{39} \mathrm{CNS}$ side-effects were more observed in higher dolutegravir trough concentrations with a median trough of $1.34 \mu \mathrm{g} / \mathrm{mL} .{ }^{39}$ Monitoring dolutegravir

Table 2 Risk ratios of neurotoxicities excluding efavirenz RCTs

\begin{tabular}{|c|c|c|c|c|c|c|}
\hline Adverse event & Drug(s) & Risk ratio & $95 \% \mathrm{Cl}$ & $P$ value & Studies included & $\mathrm{I}^{2} \%$ \\
\hline \multirow[t]{2}{*}{ Depression } & Dolutegravir & 1.26 & 0.96 to 1.65 & 0.09 & 8 & 0 \\
\hline & Dolutegravir/rilpivirine & 2.82 & 1.12 to 7.07 & 0.02 & 1 & $\mathrm{~N} / \mathrm{A}$ \\
\hline \multirow[t]{3}{*}{ Anxiety } & Dolutegravir & 0.99 & 0.72 to 1.36 & 0.95 & 7 & 12 \\
\hline & Dolutegravir/rilpivirine & 1.37 & 0.56 to 3.38 & 0.49 & 1 & $\mathrm{~N} / \mathrm{A}$ \\
\hline & Combined & 1.02 & 0.77 to 1.35 & 0.91 & 8 & 4 \\
\hline Insomnia & Combined & 1.2 & 0.93 to 1.56 & 0.16 & 10 & 23 \\
\hline \multirow[t]{3}{*}{ Dizziness } & Dolutegravir & 1.12 & 0.85 to 1.48 & 0.43 & 6 & 0 \\
\hline & Dolutegravir/rilpivirine & 20.92 & 1.23 to 356.03 & 0.04 & 1 & N/A \\
\hline & Combined & 1.23 & 0.94 to 1.62 & 0.14 & 7 & 22 \\
\hline Suicidal behaviour & Dolutegravir & 1.01 & 0.55 to 1.86 & 0.98 & 8 & 5 \\
\hline
\end{tabular}

N/A, not applicable; RCTs, randomised controlled trials. 
Table 3 Risk ratios of neurotoxicities including only efavirenz RCTs

\begin{tabular}{|c|c|c|c|c|c|c|}
\hline Adverse event & Drug(s) & Risk ratio & $95 \% \mathrm{Cl}$ & $P$ value & Studies included & $\mathrm{I}^{2} \%$ \\
\hline \multirow[t]{3}{*}{ Depression } & Dolutegravir & 0.86 & 0.56 to 1.33 & 0.51 & 1 & $\mathrm{~N} / \mathrm{A}$ \\
\hline & Rilpivirine & 1.08 & 0.80 to 1.48 & 0.22 & 4 & 33 \\
\hline & Combined & 1.02 & 0.8 to 1.48 & 0.88 & 5 & 21 \\
\hline \multirow[t]{3}{*}{ Anxiety } & Dolutegravir & 0.9 & 0.58 to 1.41 & 0.66 & 1 & $\mathrm{~N} / \mathrm{A}$ \\
\hline & Rilpivirine & 0.68 & 0.35 to 1.34 & 0.27 & 3 & 75 \\
\hline & Combined & 0.75 & 0.48 to 1.18 & 0.21 & 4 & 64 \\
\hline \multirow[t]{3}{*}{ Insomnia } & Dolutegravir & 1.07 & 0.38 to 2.99 & 0.89 & 2 & 45 \\
\hline & Rilpivirine & 1 & 0.71 to 1.41 & 1 & 4 & 62 \\
\hline & Combined & 1.06 & 0.78 to 1.44 & 0.71 & 6 & 62 \\
\hline \multirow[t]{3}{*}{ Dizziness } & Dolutegravir & 0.28 & 0.21 to 0.38 & $<0.00001$ & 2 & 0 \\
\hline & Rilpivirine & 0.35 & 0.28 to 0.43 & $<0.00001$ & 5 & 0 \\
\hline & Combined & 0.33 & 0.28 to 0.39 & $<0.00001$ & 7 & 15.3 \\
\hline \multirow[t]{3}{*}{ Suicidal behaviour } & Dolutegravir & 0.83 & 0.26 to 2.61 & 0.74 & 2 & 0 \\
\hline & Rilpivirine & 1.32 & 0.36 to 4.8 & 0.67 & 3 & 17 \\
\hline & Combined & 1 & 0.45 to 2.22 & 0.6 & 5 & 0 \\
\hline
\end{tabular}

N/A, not applicable; RCTs, randomised controlled trials.

trough concentrations around $1.34 \mu \mathrm{g} / \mathrm{mL}$ could potentially aid and reduce neurotoxic side-effects. Another postulated toxicity effect is due to a potential drug-drug interaction at breast cancer resistance protein (BCRP). BCRP is a major efflux transporter protein that removes drugs from inside cells. ${ }^{40}$ Dolutegravir is a substrate of BCRP and rilpivirine is an inhibitor of BCRP. ${ }^{41-46}$ Therefore, rilpivirine through BCRP inhibition may potentially increase the concentration of dolutegravir. ${ }^{40}$ Further studies are needed to explore this interaction.

The meta-analysis demonstrated a synergistic effect on depressive symptoms for dolutegravir/rilpivirine $(\mathrm{RR}=2.82 ; 95 \% \mathrm{CI}$ (1.12 to 7.10$)$ ) when compared with dolutegravir $(\mathrm{RR}=1.10$; $95 \% \mathrm{CI}(0.88$ to 1.38$))$ and rilpivirine $(\mathrm{RR}=1.08 ; 95 \% \mathrm{CI}(0.80$ to 1.48)). There were two significant outcomes in the primary analysis under dizziness for dolutegravir and rilpivirine. No other significant values were observed in the other defined neurotoxicities when comparing against ARV comparators. A subanalysis was conducted to remove studies that contained efavirenzbased regimens. Efavirenz has been linked to abnormal dreams, insomnia, depression and suicidal ideation. ${ }^{14}$ Due to efavirenz's side-effect profile, its frequent use has declined. It was theorised that since efavirenz has well-documented reports of neurotoxicity, it may have skewed the analysis therefore prompting an additional subanalysis. There were a few changes observed in the RR analysis of depression. The combined drug analysis RR changed from $(\mathrm{RR}=1.13,95 \% \mathrm{CI}(0.94$ to 1.36$), \mathrm{p}=0.19)$ to

Table 4 Discontinuation rates of included RCTs

\begin{tabular}{|c|c|c|c|}
\hline Study name & $\begin{array}{l}\text { Drug observed and number of } \\
\text { participants in study arm }\end{array}$ & $\begin{array}{l}\text { Discontinuation due to an adverse } \\
\text { event }\end{array}$ & Reason for discontinuation \\
\hline Cahn et $a l^{25}$ & Dolutegravir $(n=357)$ & $9 / 357(3 \%)$ & $\mathrm{N} / \mathrm{A}$ \\
\hline Dooley et $a^{27}$ & Dolutegravir $(\mathrm{n}=69)$ & $\mathrm{N} / \mathrm{A}$ & $\mathrm{N} / \mathrm{A}$ \\
\hline Molina et $a^{\beta 3}$ & Dolutegravir $(n=242)$ & $6 / 242(3.3 \%)$ & $\mathrm{N} / \mathrm{A}$ \\
\hline Molina et $a l^{24}$ & Dolutegravir $(\mathrm{n}=281)$ & $2 / 281(1 \%)$ & $\begin{array}{l}\text { Included headache }(n=1) \text { and pruritus }(n=1) \text { in the } \\
\text { dolutegravir group }\end{array}$ \\
\hline NCT00540449 (ECHO) & Rilpivirine $(n=346)$ & $6 / 346(1.7 \%)$ & N/A \\
\hline NCT01263015 (SINGLE) & Dolutegravir $(n=414)$ & $\mathrm{N} / \mathrm{A}$ & $\mathrm{N} / \mathrm{A}$ \\
\hline NCT01709084 (SALIF) & Rilpivirine ( $n=213$ ) & $\mathrm{N} / \mathrm{A}$ & $\mathrm{N} / \mathrm{A}$ \\
\hline NCT02227238 & Dolutegravir $(n=314)$ & $\mathrm{N} / \mathrm{A}$ & $\mathrm{N} / \mathrm{A}$ \\
\hline NCT02607930 & Dolutegravir $(n=315)$ & $\mathrm{N} / \mathrm{A}$ & $\mathrm{N} / \mathrm{A}$ \\
\hline Raffi et $a^{22}$ & Dolutegravir $(n=411)$ & $10 / 411(2.4 \%)$ & $\mathrm{N} / \mathrm{A}$ \\
\hline Stellbrink et $\left.a\right|^{21}$ & Dolutegravir $(n=51)$ & $1 / 51(2 \%)$ & Lipoatrophy \\
\hline Trottier et $a \beta^{30}$ & Dolutegravir $(n=276)$ & $11 / 276(4 \%)$ & $\mathrm{N} / \mathrm{A}$ \\
\hline Vera et $a l^{17}$ & Rilpivirine ( $n=37$ ) & $\mathrm{N} / \mathrm{A}$ & $\mathrm{N} / \mathrm{A}$ \\
\hline Wilkins et $a l^{16}$ & Rilpivirine ( $n=394)$ & $10 / 394(2.5 \%)$ & $\mathrm{N} / \mathrm{A}$ \\
\hline
\end{tabular}

N/A, not applicable; RCTs, randomised controlled trials. 
$(\mathrm{RR}=1.34,95 \% \mathrm{CI}$ (1.04 to 1.74), $\mathrm{p}=0.02)$. While the changes were statistically significant, it does not appear to be clinically relevant. Dolutegravir alone shared a similar trend with its RRs when excluding efavirenz changing from $(R R=1.10$, $95 \%$ CI (0.88 to 1.38$)$ ) to ( $R R=1.26,95 \%$ CI (0.96 to 1.65$)$, $\mathrm{p}=0.09)$. The analysis and the subanalysis indicate that dolutegravir may contribute to the neurotoxic adverse effects along with rilpivirine. Practitioners should not only exercise caution when prescribing the combination of dolutegravir/rilpivirine to patients with a medical history of CNS abnormalities but any ART containing either dolutegravir or rilpivirine.

There were several limitations to this meta-analysis. The metaanalysis was not sufficiently powered due to using RCTs that were testing efficacy and not adverse drug-related events. Out of the 20 studies initially identified, only 15 reported adverse effects of depression relative to the meta-analysis' primary outcome. Dolutegravir/rilpivirine only had one RCT included. As of August 2020, there are two RCTs observing dolutegravir/ rilpivirine use that are either in the recruiting phase or have been completed; however, the results are not currently available. ${ }^{478}$

\section{CONCLUSION}

In this meta-analysis of 20 randomised trials, there was a synergistic risk of depression when dolutegravir was used in combination with rilpivirine. Despite the increase, the clinical significance is unknown and needs further study. Additionally, neuropsychiatric adverse effect risk appears similar between dolutegravir and rilpivirine ART when compared with efavirenz-based ART. The combination of dolutegravir and rilpivirine has not been directly compared with efavirenz and needs additional trials.

\section{Key messages}

- Dolutegravir may contribute to the neuropsychiatric adverse effects of the combination of dolutegravir/rilpivirine used in HIV.

- The meta-analysis included 20 randomised controlled trials that observed neuropsychiatric adverse effects focusing on depression, anxiety, insomnia, dizziness and suicidal behaviour.

- The combination of dolutegravir/rilpivirine demonstrated a synergistic effect for depressive symptoms. Despite the increase, the clinical implications are not clear and need additional research. None of the other observed neuropsychiatric adverse effects were increased.

\section{Handling editor Laith J Abu-Raddad}

Contributors AAR developed the research question and methods section, and developed the search strategy. AAR and AVF selected eligible studies during the title/ abstract screening and full-text evaluation. AAR and AVF extracted data from the included studies and appraised the quality of the evidence. JC, JET and JFM reviewed data extraction and appraisal of evidence. AAR and CH coordinated RevMan V.5.3 implementation. AAR entered extracted data into RevMan V.5.3 and interpreted/ analysed the data. AVF, JC, JET and JFM double-checked data entry into RevMan V.5.3 and reviewed data interpretation. CH was involved in cases of dissent with respect to extracted data/evaluations, and participated in analysing and interpreting the data. AAR developed the draft of the manuscript. AVF, JC, JET, JFM and CH contributed to the final editing of the draft. AAR and JC coordinated contributions of the coauthors and compiled the final draft.

Funding The authors have not declared a specific grant for this research from any funding agency in the public, commercial or not-for-profit sectors.

Competing interests None declared.

Patient consent for publication Not required.

Provenance and peer review Not commissioned; externally peer reviewed.
Supplemental material This content has been supplied by the author(s). It has not been vetted by BMJ Publishing Group Limited (BMJ) and may not have been peer-reviewed. Any opinions or recommendations discussed are solely those of the author(s) and are not endorsed by BMJ. BMJ disclaims all liability and responsibility arising from any reliance placed on the content. Where the content includes any translated material, BMJ does not warrant the accuracy and reliability of the translations (including but not limited to local regulations, clinical guidelines, terminology, drug names and drug dosages), and is not responsible for any error and/or omissions arising from translation and adaptation or otherwise.

\section{ORCID iD}

Anthony Allen Reeves http://orcid.org/0000-0002-1739-948X

\section{REFERENCES}

1 Global HIV \& AIDS statistics - 2020 fact sheet. UNAIDS (2020). Available: https:/l www.unaids.org/en/resources/fact-sheet [Accessed 30 Jul 2020].

2 Bezabhe WM, Chalmers L, Bereznicki LR, et al. Adherence to antiretroviral therapy and virologic failure: a meta-analysis. Medicine 2016;95:e3361.

3 Tyree GA, Vaida F, Zisook S, et al. Clinical correlates of depression chronicity among people living with HIV: what is the role of suicidal ideation? I Affect Disord 2019:258:163-71.

4 Treisman GJ, Soudry O. Neuropsychiatric effects of HIV antiviral medications. Drug Saf 2016:39:945-57

5 Panel on Antiretroviral Guidelines for Adults and Adolescents. Guidelines for the Use of Antiretroviral Agents in Adults and Adolescents with HIV. Department of Health and Human Services. Available: http://aidsinfo.nih.gov/contentfiles/lvguidelines/ AdultandAdolescentGL.pdf [Accessed 1 Aug 2020].

$6 \mathrm{Ma}$ Q, Schifitto G, Venuto C, et al. Effect of dolutegravir and sertraline on the blood brain barrier (BBB). J Neuroimmune Pharmacol 2020;15:7-9.

7 Tivicay (dolutegravir) [prescribing information]. Research Triangle Park, NC: ViiV Healthcare 2020

8 Edurant (rilpivirine) [prescribing information]. Titusville, NJ: Janssen Therapeutics 2019.

9 Juluca (dolutegravir/rilpivirine) [prescribing information]. Research Triangle Park, NJ: ViiV Healthcare 2019.

10 Ryan R, Dayaram YK, Schaible D, et al. Outcomes in older versus younger patients over 96 weeks in HIV-1- infected patients treated with rilpivirine or efavirenz in echo and thrive. Curr HIV Res 2013;11:570-5.

11 HIVIAIDS Glossary Backbone Definition. National Institutes of health, 2020. Available: https://aidsinfo.nih.gov/understanding-hiv-aids/glossary/4595/backbone [Accessed 16 Apr 2020].

12 Jadad AR, Moore RA, Carroll D, et al. Assessing the quality of reports of randomized clinical trials: is blinding necessary? Control Clin Trials 1996;17:1-12.

13 Seden K, Kiiza D, Laker E, et al. High prevalence and long duration of nervous system and psychiatric adverse drug reactions in Ugandan patients taking efavirenz $600 \mathrm{mg}$ daily. J Antimicrob Chemother 2018;73:3158-61.

14 Sustiva (efavirenz) [prescribing information]. Princeton, NJ: Bristol Myers Squibb 2019.

15 Nordic Cochrane Centre. Review manager 5 (RevMan 5). version 5.3. Copenhagen: Nordic Cochrane Centre, The Cochrane Collaboration, 2014.

16 Wilkins EL, Cohen CJ, Trottier B, et al. Patient-Reported outcomes in the single-tablet regimen (StAR) trial of rilpivirine/emtricitabine/tenofovir disoproxil fumarate versus efavirenz/emtricitabine/tenofovir disoproxil fumarate in antiretroviral treatmentnaive adults infected with HIV-1 through 48 weeks of treatment. AIDS Care 2016;28:401-8.

17 Vera JH, Bracchi M, Alagaratnam J, et al. Improved central nervous system symptoms in people with HIV without objective neuropsychiatric complaints switching from efavirenz to rilpivirine containing CART. Brain Sci 2019:9:195

18 ClinicalTrials.gov [Internet]. Bethesda (MD): National library of medicine (US). 2000 Feb 29 -. identifier NCT01709084, a clinical trial comparing the efficacy of tenofovir disoproxil Fumarate/Emtricitabine/Rilpivirine (TDF/FTC/RPV) versus TDF/FTC/Efavirenz (TDF/FTC/EFV) in patients with undetectable plasma HIV-1 RNA on current first-line treatment (SALIF); 2012 October 17. Available: https://clinicaltrials.gov/ct2/show/ study/NCT01709084?term=rilpivirine\&rslt=With\&draw=2\&rank=4 [Accessed $17 \mathrm{Apr}$ 2020].

19 ClinicalTrials.gov [Internet]. Bethesda (MD): National library of medicine (US). 2000 Feb 29 -. identifier NCT00540449, a clinical trial in treatment naive HIV-1 patients comparing TMC278 to efavirenz in combination with tenofovir + emtricitabine. (echo); 2007 October 8. Available: https://clinicaltrials.gov/ct2/show/study/ NCT00540449?term=rilpivirine\&rslt=With\&draw=1 [Accessed 17 Apr 2020]

20 ClinicalTrials.gov [Internet]. Bethesda (MD): National library of medicine (US). 2000 Feb 29 -. identifier NCT00543725, TMC278-TiDP6-C215: a clinical trial in treatment naive HIV-subjects patients comparing TMC278 to efavirenz in combination with 2 nucleoside/nucleotide reverse transcriptase inhibitors (thrive); 2007 October 15. Available: https://clinicaltrials.gov/ct2/show/study/NCT00543725 [Accessed 17 Apr 2020].

21 Stellbrink H-J, Reynes J, Lazzarin A, et al. Dolutegravir in antiretroviral-naive adults with HIV-1: 96-week results from a randomized dose-ranging study. AIDS 2013;27:1771-8 
22 Raffi F, Jaeger H, Quiros-Roldan E, et al. Once-Daily dolutegravir versus twice-daily raltegravir in antiretroviral-naive adults with HIV-1 infection (SPRING-2 study): 96 week results from a randomised, double-blind, non-inferiority trial. Lancet Infect Dis 2013;13:927-35.

23 A Trial Comparing GSK1349572 50mg Plus Abacavir/Lamivudine Once Daily to Atripla (Also Called The SINGLE Trial) - Full Text View. Full Text View - ClinicalTrials.gov. Available: https://clinicaltrials.gov/ct2/show/NCT01263015 [Accessed 27 Dec 2020].

24 Molina J-M, Clotet B, van Lunzen J, et al. Once-Daily dolutegravir versus darunavir plus ritonavir for treatment-naive adults with HIV-1 infection (flamingo): 96 week results from a randomised, open-label, phase 3B study. Lancet HIV 2015;2:e127-36.

25 Cahn P, Pozniak AL, Mingrone H, et al. Dolutegravir versus raltegravir in antiretroviralexperienced, integrase-inhibitor-naive adults with HIV: week 48 results from the randomised, double-blind, non-inferiority SAILING study. Lancet 2013;382:700-8.

26 Orrell C, Hagins DP, Belonosova E, et al. Fixed-Dose combination dolutegravir, abacavir, and lamivudine versus ritonavir-boosted atazanavir plus tenofovir disoproxil fumarate and emtricitabine in previously untreated women with HIV-1 infection (ARIA): week 48 results from a randomised, open-label, non-inferiority, phase 3B study . Lancet HIV 2017;4:e536-46.

27 Dooley KE, Kaplan R, Mwelase N. International study of patients with HIV on rifampicin ING Study Group. Dolutegravir-based antiretroviral therapy for patients coinfected with tuberculosis and human immunodeficiency virus: a multicenter, Noncomparative, open-label, randomized trial. Clin Infect Dis 2020;70:549-56.

28 Sax PE, DeJesus E, Crofoot G, et al. Bictegravir versus dolutegravir, each with emtricitabine and tenofovir alafenamide, for initial treatment of HIV-1 infection: a randomised, double-blind, phase 2 trial. Lancet HIV 2017;4:e154-60.

29 ClinicalTrials.gov [Internet]. Bethesda (MD): National library of medicine (US). 2000 Feb 29 -. identifier NCT02607956, safety and efficacy of Bictegravir/ Emtricitabine/Tenofovir Alafenamide versus dolutegravir + Emtricitabine/Tenofovir Alafenamide in HIV-1 infected, antiretroviral treatment-naive adults; 2015 November 18. Available: https://clinicaltrials.gov/ct2/show/study/NCT02607956? term $=$ dolutegravir\&rs $\mid t=$ With\&type $=$ Intr\&age $=12 \& \mathrm{draw}=2 \&$ rank $=18$ [Accessed 17 Apr 2020].

30 Trottier B, Lake JE, Logue K, et al. Dolutegravir/abacavir/lamivudine versus current art in virally suppressed patients (STRIIVING): a 48-week, randomized, non-inferiority, open-label, phase IIIB study. Antivir Ther 2017;22:295-305.

31 ClinicalTrials.gov [Internet]. Bethesda (MD): National library of medicine (US). 2000 Feb 29 -. identifier NCT02227238; comparative efficacy and safety study of dolutegravir and Lopinavir/Ritonavir in second-line treatment; 2014 August 28. Available: https://clinicaltrials.gov/ct2/show/NCT02227238 [Accessed 17 Apr 2020].

32 ClinicalTrials.gov [Internet]. Bethesda (MD): National library of medicine (US). 2000 Feb 29 -. identifier NCT03110380; switching to a fixed dose combination of Bictegravir/Emtricitabine/Tenofovir Alafenamide (B/F/TAF) in HIV-1 infected adults who are virologically suppressed; 2017 April 12. Available: https://clinicaltrials.gov/ ct2/show/NCT03110380 [Accessed 17 Apr 2020].

33 Molina J-M, Ward D, Brar I, et al. Switching to fixed-dose bictegravir, emtricitabine, and tenofovir alafenamide from dolutegravir plus abacavir and lamivudine in virologically suppressed adults with HIV-1: 48 week results of a randomised, double-blind, multicentre, active-controlled, phase 3, non-inferiority trial. Lancet HIV 2018;5:e357-65.

34 ClinicalTrials.gov [Internet]. Bethesda (MD): National library of medicine (US). 2000 Feb 29 -. identifier NCT02607930; safety and efficacy of Bictegravir/Emtricitabine/ Tenofovir Alafenamide versus Abacavir/Dolutegravir/Lamivudine in HIV-1 infected, antiretroviral treatment-naïve adults; 2015 November 18. Available: https:// clinicaltrials.gov/ct2/show/NCT02607930 [Accessed 17 Apr 2020].

35 Llibre JM, Hung C-C, Brinson C, et al. Efficacy, safety, and tolerability of dolutegravirrilpivirine for the maintenance of virological suppression in adults with HIV-1: phase 3, randomised, non-inferiority SWORD-1 and SWORD-2 studies. Lancet 2018;391:839-49.

36 Letendre SL, Mills AM, Tashima KT, et al. ING116070: a study of the pharmacokinetics and antiviral activity of dolutegravir in cerebrospinal fluid in HIV-1-infected, antiretroviral therapy-naive subjects. Clin Infect Dis 2014;59:1032-7.

37 Thibaut G, Valérie F, Anne-Marie T. Dolutegravir cerebrospinal fluid diffusion in HIV-1Infected patients with central nervous system impairment. Open Forum Infectious Diseases 2019;6.

38 Montenegro-Burke JR, Woldstad CJ, Fang M, et al. Nanoformulated antiretroviral therapy attenuates brain metabolic oxidative stress. Mol Neurobio/ 2019;56:2896-907.

39 Yagura H, Watanabe D, Nakauchi T. Effect of dolutegravir plasma concentration on central nervous system side effects. Seattle, Washington: Poster present at: Conference on retroviruses and opportunistic infections, 2017. https://www. croiconference.org/abstract/effect-dolutegravir-plasma-concentration-central-nervoussystem-side-effects/\#: :text=At $\% 20$ least $\% 20$ one $\% 20$ of $\% 20$ the,anxiety $\% 20 \% 5 B 3 \%$ 20(7\%25)\%5D

40 Lee CA, O'Connor MA, Ritchie TK, et al. Breast cancer resistance protein (ABCG2) in clinical pharmacokinetics and drug interactions: practical recommendations for clinical victim and perpetrator drug-drug interaction study design. Drug Metab Dispos 2015:43:490-509.

41 Gutiérrez F, Fulladosa X, Barril G, et al. Renal tubular transporter-mediated interactions of HIV drugs: implications for patient management. AIDS ReV 2014;16)::199-212.

42 Moss DM, Liptrott NJ, Curley P, et al. Rilpivirine inhibits drug transporters ABCB1, SLC22A1, and SLC22A2 in vitro. Antimicrob Agents Chemother 2013;57:5612-8.

43 Reznicek J, Ceckova M, Ptackova Z, et al. Mdr1 and BCRP transporter-mediated drugdrug interaction between rilpivirine and abacavir and effect on intestinal absorption. Antimicrob Agents Chemother 2017;61:e00837-17.

44 Reese MJ, Savina PM, Generaux GT, et al. In vitro investigations into the roles of drug transporters and metabolizing enzymes in the disposition and drug interactions of dolutegravir, a HIV integrase inhibitor. Drug Metab Dispos 2013;41:353-61.

45 Cottrell ML, Hadzic T, Kashuba ADM. Clinical pharmacokinetic, pharmacodynamic and drug-interaction profile of the integrase inhibitor dolutegravir. Clin Pharmacokinet 2013:52:981-94

46 National Center for Biotechnology Information. PubChem compound summary for Cid 54726191, dolutegravir. Available: https://pubchem.ncbi.nlm.nih.gov/compound/ Dolutegravir [Accessed 25 Dec 2020].

47 ClinicalTrials.gov [Internet]. Bethesda (MD): National Library of Medicine (US). 2000 Feb 29 -. Identifier NCT03518060, Dolutegravir/Rilpivirine, Antiretroviral Efficacy Study Using Real-world Data in Subjects With Human Immunodeficiency Virus (HIV)-1 (JUNGLE); 2018 May 8. Available: https://clinicaltrials.gov/ct2/show/NCT03518060 [Accessed 17 Apr 2020].

48 ClinicalTrials.gov [Internet]. Bethesda (MD): National Library of Medicine (US). 2000 Feb 29 -. Identifier NCT04019873, 'COMBINE-2': Real-world Evidence for Effectiveness of Two Drug Regimen, Antiretroviral Therapy With Integrase Inhibitors Plus a Reverse Transcriptase Inhibitor; 2019 July 15. Available: https://clinicaltrials. gov/ct2/show/NCT04019873 [Accessed 17 Apr 2020]. 